Celal Bayar University Journal of Science

\title{
Analysis of a Capacitor Modelled with Conformable Fractional Derivative under DC and Sinusoidal Signals
}

\author{
Utku Palaz ${ }^{1 *(D)}$, Reşat Mutlu 2 iD \\ ${ }^{1}$ Ministry of Education of Republic of Turkey \\ ${ }^{2}$ Department of Electronics and Telecommunication Engineering, Namık Kemal University, Çorlu, Tekirdağ, \\ Turkey \\ *utkupalaz@gmail.com \\ *Orcid: 0000-0003-4579-0424
}

Received: 25 June 2020

Accepted: 1 June 2021

DOI: $10.18466 /$ cbayarfbe. 757813

\begin{abstract}
Fractional order circuit elements are successfully used to model circuits and systems in the last few decades. There are different types of fractional derivatives. Recently, another one named "the conformable fractional derivative" (CFD) has been introduced and shown to give good results for modeling supercapacitors. It is imperative to know how circuit elements behave for different current and voltage waveforms in circuit theory so that they can be exploited at their full potential. A CFD capacitor is not a well-known element, and its usage and circuit solutions are rarely addressed in the literature. In this study, it is examined how a CFD capacitor would behave under DC and AC excitations when it is fed by not only a current source but also a voltage source.
\end{abstract}

Keywords: Fractional Order Derivatives, Circuit Analysis, Circuit Theory, Energy Analysis, Circuit Modeling

\section{Introduction}

Fractional derivative (FD) is a branch of mathematical analysis [1,2]. A differential operator can be of any arbitrary order within it. It has first appeared in the $17^{\text {th }}$ century [3]. In the last decades, Fractional Calculus has emerged as a popular research area because of its applicability in many different fields [4-5]. The selftaught Oliver Heaviside has used fractional calculus to find the solution of the telegrapher's equation around 1890 [3]. The fractional-order circuit elements are used to model circuit elements such as capacitors, inductors and memristors [6-10]. Filters, controllers and oscillators which are based on fractional-order circuit elements are made or can be used to model systems [45, 8-9, 11-14]. Another FD is suggested in [15]. It is named as "the conformable fractional derivative" (CFD). Its definition is built on the conventional limit definition of the derivative of a function. It casts off the other FD definitions [15]. This makes it simpler and advantageous than the other FDs. The CFD is elaborated in [16]. However, a CFD is actually not an FD: it can be described as a first-order derivative time a power function of the independent variable $[15,16]$. This new definition is a broadening of the ordinary derivative. It is also distinct from the other FDs. The CFD is able to accommodate the common features of
FDs. Well-known calculus theorems such as product rule, Rolle's Theorem, Average Value Theorem, partial integration, Taylor series can be easily extended or applied to the CFD. The conformal fractional derivative has a very important property: while the Riemann-Liouville FD of a constant is not zero, the CFD of a constant is zero. Due to these properties, the conformal fractional derivative has become a hot research area. The conformal derivative has also the advantage of being physically interpretable compared to the other types of fractional derivatives [17]. Usage of the FDs in electric circuits has been examined in [18]. Supercapacitors have been modelled using fractionalorder models in [19-22]. The oscillation of impulsive conformable fractional differential equations has been inspected in [23]. Electric circuits modelled with FD circuit elements under sinusoidal excitation have been analyzed with the enhanced fractional derivative method, which is called Caputo FD generalizing the differential equations and ordinary integrals are not necessary to describe the fractional-order initial conditions like Riemann-Liouville FD [24-25]. Several electric circuits characterized by CFDs have been solved in [26]. Other circuits modelled with the CFD have been examined in [27]. The conformable fractional derivative has been used to analyze an electric circuit containing a supercapacitor in [28]. Analytical solutions of electrical circuits described by fractional conformable 
derivatives in Liouville-Caputo sense is given in [29]. Electric circuits of the CFDs with and without singular kernels have been solved in [30].

It is important to analyze new circuit elements for different current and voltage waveforms so that they can be exploited at full potential. In this paper, the conformal fractional derivative capacitor model for DC and sinusoidal waveforms have been solved. CFD capacitor has been fed with not only voltage sources but also for current sources. The analytical solutions were given with incomplete gamma function for sinusoidal voltage case. The discussions are provided in the conclusion section.

The rest of the paper is structured as follows. The CFD capacitor model is given in the second section. Its analysis for DC and AC signals are given in the third section. Op-amp-based differentiator and integrator circuits with a CFD capacitor are examined in the fourth section. Finally, the paper is concluded in "Conclusions" section.

\section{Conformal Fractional Derivative and CFD Capacitor Constitutional Law}

The CFD is described in [15] as the follows:

Definition 1. Let $f:[0,+\infty) \rightarrow R$ and $t>0$. The CFD for $0<\alpha \leq 1$ is described as

$$
D_{\alpha} f(t)=\lim _{p \rightarrow 0} \frac{f\left(t+p^{(1-\alpha)}\right)-f(t)}{p}
$$

For $\mathrm{t}>0$ and the conformable fractional derivative at 0 is defined as $D_{\alpha} f(0)=\lim _{t \rightarrow 0^{+}}\left(D_{\alpha} f\right)(t)$. If it is differentiable then $D_{\alpha} f(t)=t^{1-\alpha} f^{\prime}(t)$

Definition 2. Let $\alpha \in(0,1]$. The conformable fractional integral of a function $f:[0,+\infty) \rightarrow R$ of order a is denoted by $I_{\alpha} f(t)$ and is defined as

$$
\begin{aligned}
I_{\alpha} f(t) & =\int_{0}^{t} s^{(\alpha-1)} f(s) . d s \\
\frac{d^{\alpha} f(t)}{d t^{\alpha}} & =f^{\prime}(t) t^{1-\alpha}=\frac{d f(t)}{d t} t^{1-\alpha}
\end{aligned}
$$

More information about CFD can be found in [15-17]. If a capacitor can be modelled using CFD [28], its constitutive law can be written as

$$
i_{C}(t)=C_{\alpha} \frac{d^{\alpha} v_{C}(t)}{d t^{\alpha}}
$$

Where $i_{C}(t), v_{C}(t)$ and $C_{\alpha}$ are CFD capacitor current, CFD capacitor voltage and CFD capacitor coefficient, respectively.

\section{The CFD Capacitor Fed by a Current Source}

In this section, the solutions of a CFD capacitor is found if it is fed by a current source as shown in Figure 1 for the cases: (a) the current source being constant and (b) it being sinusoidal.

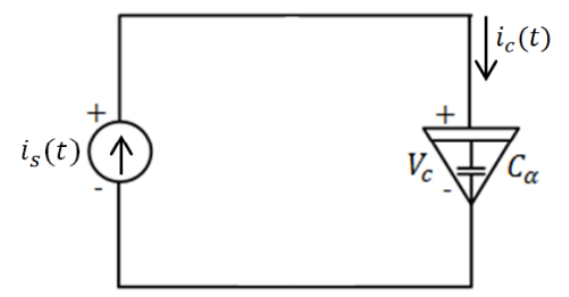

Figure 1: The CFD capacitor fed by a current source

\subsection{Constant Current Solution}

If the current source has a constant magnitude or the CFD capacitor current is constant;

$$
i_{s}(t)=i_{c}(t)=I_{d c}=C_{\alpha} \frac{d v_{C}(t)}{d t} t^{1-\alpha}
$$

By arranging both sides,

$$
\frac{d v_{C}(t)}{d t}=\frac{I_{d c}}{C_{\alpha} t^{1-\alpha}} \rightarrow d v_{C}(t)=\frac{I_{d c}}{C_{\alpha} t^{1-\alpha}} d t
$$

Its voltage can be found as

$$
\begin{gathered}
v_{C}(t)=\int \frac{I_{d c}}{C_{\alpha} t^{1-\alpha}} d t=\frac{I_{d c}}{C_{\alpha}} \int t^{\alpha-1} d t \\
v_{C}(t)=\frac{I_{d c} t^{\alpha}}{C_{\alpha} \alpha}+K
\end{gathered}
$$

Where $\mathrm{K}$ is the integration constant and the capacitor voltage at $t=0$ is used to find $\mathrm{K}$;

$$
v_{C}(0)=\frac{I_{d c} 0^{\alpha}}{C_{\alpha} \alpha}+K \rightarrow K=v_{C}(0)
$$

Using the integration constant, the CFD capacitor current can be obtained as;

$$
v_{C}(t)=\frac{I_{d c} t^{\alpha}}{C_{\alpha} \alpha}+v_{C}(0)
$$

\subsection{Sinusoidal Current Solution}

If a sinusoidal current of $i_{s}(t)=I_{m} \cos (\omega t+\varphi)$ is applied to the CFD capacitor, its voltage can be calculated with the following steps: 
$i_{s}(t)=i_{c}(t)=I_{m} \cos (\omega t+\varphi)=C_{\alpha} \frac{d v_{C}(t)}{d t} t^{1-\alpha}$

By arranging both sides of the equation, the CFD capacitor voltage is written as

$$
\begin{aligned}
& \frac{d v_{C}(t)}{d t}=\frac{I_{m} \cos (\omega t+\varphi)}{C_{\alpha} t^{1-\alpha}} \\
& v_{c}(t)=\frac{I_{m}}{C_{\alpha}} \int \cos (\omega t+\varphi) t^{(\alpha-1)} d t
\end{aligned}
$$

Using Wolfram Alpha [31], the solution of the integral is found as

$$
\nu_{c}(t)=\frac{I_{m}}{C_{\alpha}}\left(\begin{array}{l}
-\frac{t^{\alpha}\left(\omega^{2} t^{2}\right)^{-\alpha}}{2}\left((-i \omega t)^{\alpha}(\cos (\varphi)-i \sin (\varphi)) \Gamma(a, i \omega t)+\right. \\
\left.(i \omega t)^{\alpha}(\cos (\varphi)+i \sin (\varphi)) \Gamma(a,-i \omega t)\right)+K
\end{array}\right)
$$

Using the following identities:

$$
e^{i \varphi}=\cos (\varphi)+i \sin (\varphi)
$$$$
e^{-i \varphi}=\cos (\varphi)-i \sin (\varphi)
$$

The capacitor voltage turns into;

$$
v_{c}(t)=\frac{I_{m}}{C_{\alpha}}\left(\begin{array}{l}
-\frac{t^{\alpha}\left(\omega^{2} t^{2}\right)^{-\alpha}}{2}\left((-i \omega t)^{\alpha} e^{-i \varphi}\right) \Gamma(a, i \omega t)+ \\
\left.\left.(i \omega t)^{\alpha} e^{i \varphi}\right) \Gamma(a,-i \omega t)\right)+K
\end{array}\right)
$$

In mathematics, the upper incomplete gamma function is a transcendental function that appears as solutions to diverse problems such as definite integrals.

When splitting the incomplete gamma function at $\mathrm{s}$ point $x \geq 0$, two types of the incomplete gamma functions called upper and lower are obtained.

The definition of the upper incomplete gamma function intervals are explained from $x$ to $\infty$.

$$
\Gamma(s, x)=\int_{x}^{\infty} t^{s-1} e^{-t} d t
$$

If the initial condition is used at $\mathrm{t}=0$,

$v_{c}(0)=\frac{I_{m}}{C_{\alpha}}[0+K] \rightarrow K=v_{c}(0) \frac{C_{\alpha}}{I_{m}}$

Using the integration constant, the CFD capacitor voltage can be obtained as

$$
\begin{aligned}
& v_{c}(t)=-\frac{I_{m} t^{\alpha}\left(\omega^{2} t^{2}\right)^{-\alpha}}{2 C_{\alpha}}\left((-i \omega t)^{\alpha} e^{-i \varphi}\right) \Gamma(a, i \omega t)+ \\
& \left.\left((i \omega t)^{\alpha} e^{i \varphi}\right) \Gamma(a,-i \omega t)\right)+v_{c}(0)
\end{aligned}
$$

Numerical methods can be used to calculate the CFD capacitor voltage since the solution requires the upper incomplete gamma function which is also evaluated numerically.

\section{The CFD Capacitor Fed by a Voltage Source}

In this section, the solution of the CFD capacitor fed by a voltage source shown in Figure 2 is found for both of the cases: the voltage source being a constant (a) and a sinusoidal (b).

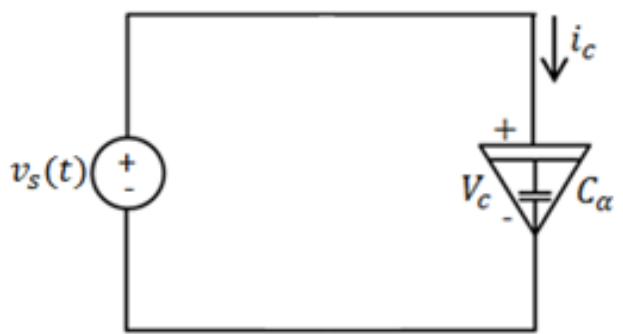

Figure 2. The CFD capacitor fed by a voltage source

\subsection{Constant Voltage Solution}

If a step function voltage is applied to the CFD capacitor at time being $t_{0}$ :

$v_{C}(t)=V_{d c} u\left(t-t_{0}\right)$

The CFD capacitor current becomes

$i_{c}(t)=C_{\alpha} \frac{d v_{C}(t)}{d t} t^{1-\alpha}=C_{\alpha} \frac{d\left(V_{d c} u\left(t-t_{0}\right)\right)}{d t} t^{1-\alpha}$

$i_{c}(t)=C_{\alpha} V_{d c} \delta\left(t-t_{0}\right) t^{1-\alpha}=C_{\alpha} V_{d c} \delta\left(t-t_{0}\right) t_{0}^{1-\alpha}$

Where $\delta\left(t-t_{0}\right)$ is the Dirac-delta function [32] shifted to time being $t_{0}$.

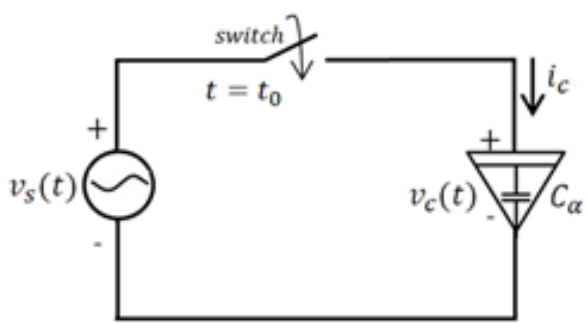

Figure 3. The CFD capacitor fed by a voltage source and depends on the time.

A CFD capacitor without a series resistor withdraws a Dirac pulse as a linear time-invariant resistor does. However, its current magnitude depends on the time the voltage is applied opposite to the case in which an LTI (Linear Time-Invariant) capacitor current pulse 
magnitude is constant not depending on the time the step function is applied as shown in Figure 3.
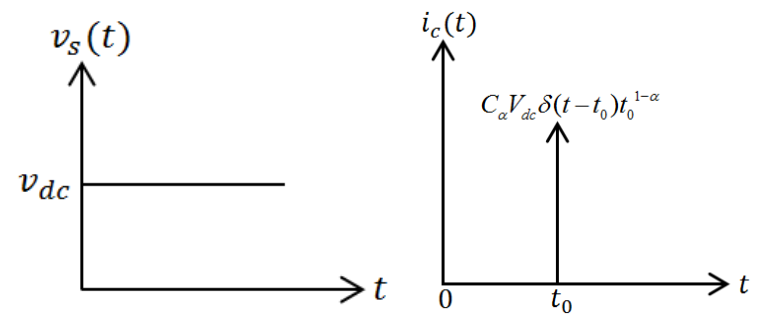

Figure 4. The CFD capacitor voltage and current vs. time when it is fed by a constant voltage

\subsection{Sinusoidal Voltage Solution}

If a sinusoidal voltage of $v_{C}(t)=V_{m} \sin (\omega t+\varphi) u\left(t-t_{0}\right)$ is applied to the CFD capacitor at time being $t_{0}$, its current is found as

$$
\begin{aligned}
& i_{c}(t)=C_{\alpha} \frac{d v_{C}(t)}{d t} t^{1-\alpha}=C_{\alpha} \frac{d\left(V_{m} \sin (\omega t) u\left(t-t_{0}\right)\right)}{d t} t^{1-\alpha} \\
& i_{c}(t)=C_{\alpha} V_{m} t^{1-\alpha}\left(\omega \cos (\omega t)+\sin (\omega t) \delta\left(t-t_{0}\right)\right)
\end{aligned}
$$

When the Dirac-delta function rule $f(t) \delta\left(t-t_{0}\right)=f\left(t_{0}\right) \delta\left(t-t_{0}\right)$ is used, the CFD capacitor current can be written as:

$$
i_{c}(t)=C_{\alpha} V_{m} \omega \cos (\omega t) t^{1-\alpha}+C_{\alpha} V_{m} \sin \left(\omega t_{0}\right) t_{0}^{1-\alpha} \delta\left(t-t_{0}\right)
$$

The circuit waveforms are drawn with Matlab ${ }^{\mathrm{TM}}$. The CFD capacitor current and voltage for this case is shown in Figure 5 and 6 . The CFD capacitor current magnitude is not constant and varies with time due to the term $t^{1-\alpha}$ as shown in Figure 4. When $V_{m}=5 \mathrm{~V}$, $\omega=1 \mathrm{rad} / \mathrm{s}, \varphi=0 \mathrm{rad} / \mathrm{s}$ and $C_{\alpha}=1 F / s^{1-\alpha}$ values are used, the CFD capacitor current graph in Figure 6 is sketched for three different alpha values.

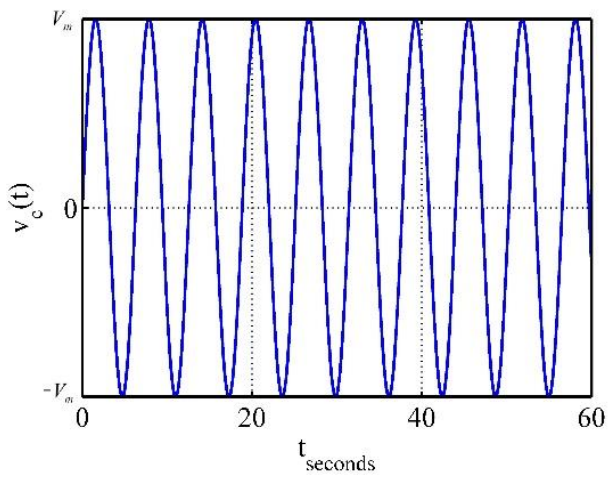

Figure 5. The CFD capacitor voltage vs. time when it is fed by a sinusoidal voltage

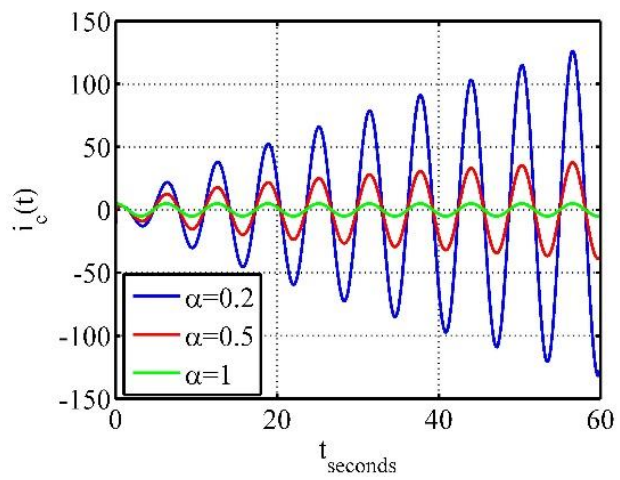

Figure 6. The CFD current vs. time when it is fed by a sinusoidal voltage

All real capacitor models have series and parallel resistors used in their equivalent circuit to model their losses. In this study, the capacitors are assumed to be ideal, ie. without losses and that's why no resistor is used in the analysis. The CFD capacitor current goes to infinity due to the term $t^{1-\alpha}$ as seen in Figure 6 because of this. Such a situation would not appear in a more realistic capacitor model when a series resistor is used.

\section{Conclusions}

Supercapacitors or ultra-capacitors are becoming more common each day. They cannot be modelled with capacitor constitutive law. Therefore, easier and robust models are needed for their usage and analysis in the circuits. Only then, such capacitors can be fully exploited.

The conformal fractional derivative provides an easier solution than the fractional derivatives such as Caputo and Riemann Liouville. Some capacitors can be modelled using fractional-order derivatives. In this study, the conformal fractional derivative is used to model a capacitor. Every circuit element should be examined for the basic waveforms such as DC and sinusoidal signals. The solutions of the CFD capacitor for the waveforms are given. Only in the case when it is fed by a current source, its voltage is found using a transcendental function the incomplete gamma functions. The solutions can be used in the analysis of the circuits with a CFD capacitor if a supercapacitor is found to be modelled with a good accuracy using experimental data. In comparison to the prior studies, the CFD derivative is more practical and analytical solutions are found to be possible while it has been not possible for other fractional derivatives. The analysis given here can also be used in modelling renewable energy systems or inverters with supercapacitors in the future. This paper can also be used as a tutorial for the researchers who have just been introduced to the research area. 


\section{Author's Contributions}

Utku Palaz: Made literature search, prepared computer codes for numerical solutions of the sample problems, made simulations, and wrote the manuscript.

Reşat Mutlu: Made literature search, assisted in analytical analysis on the structure, supervised the experiment's progress, result in interpretation, and helped in manuscript preparation.

\section{Ethics}

There are no ethical issues after the publication of this manuscript.

\section{References}

1. Podlubny, I. (1998). Fractional differential equations: an introduction to fractional derivatives, fractional differential equations to methods of their solution and some of their applications. Elsevier.

2. Yang, X. J. (2019). General fractional derivatives: theory, methods and applications. Chapman and Hall/CRC.

3. Ross, B. (1977). The development of fractional calculus 1695 1900. Historia Mathematica, 4(1), 75-89.

4. Kilbas, A.A.; Srivastava, H.M.; Trujillo, J.J. Theory and Applications of Fractional Differential Equations; Elsevier Amsterdam, The Netherlands, 2006

5. Babiarz, A., Czornik, A., Klamka, J., \& Niezabitowski, M (2017). Theory and applications of non-integer order systems. Lecture Notes Electrical Engineering, 407.

6. Moreles, M. A., \& Lainez, R. (2016). Mathematical modelling of fractional order circuits. arXiv preprint arXiv:1602.03541.

7. Freeborn, T. J. (2013). A survey of fractional-order circuit models for biology and biomedicine. IEEE Journal on emerging and selected topics in circuits and systems, 3(3), 416-424.

8. Adhikary, A., Khanra, M., Pal, J., \& Biswas, K. (2017) Realization of fractional order elements. Inae Letters, 2(2), 41-47.

9. Tsirimokou, G., Kartci, A., Koton, J., Herencsar, N., \& Psychalinos, C. (2018). Comparative study of discrete componen realizations of fractional-order capacitor and inductor active emulators. Journal of Circuits, Systems and Computers, 27(11), 1850170

10. Kartci, A., Agambayev, A., Herencsar, N., \& Salama, K. N. (2018). Series-, parallel-, and inter-connection of solid-state arbitrary fractional-order capacitors: theoretical study and experimental verification. IEEE Access, 6, 10933-10943.

11. Sotner, R., Jerabek, J., Kartci, A., Domansky, O., Herencsar, N., Kledrowetz, V., ... \& Yeroglu, C. (2019). Electronically reconfigurable two-path fractional-order PI/D controller employing constant phase blocks based on bilinear segments using CMOS modified current differencing unit. Microelectronics Journal, 86, 114 129.

12. Podlubny, I., Petráš, I., Vinagre, B. M., O'leary, P., \& Dorčák, L. (2002). Analogue realizations of fractional-order controllers. Nonlinear dynamics, 29(1-4), 281-296.

13. Alagoz, B. B., \& Alisoy, H. Z. (2014). On the Harmonic Oscillation of High-order Linear Time Invariant Systems. Scientific Committee.

14. Alagöz, B, B \& Alisoy, H. Estimation of Reduced Order Equivalent Circuit Model Parameters of Batteries from Noisy Current and Voltage Measurements. Balkan Journal of Electrical and Computer Engineering, 6(4), 224-231.

15. Khalil, R.; al Horani, M.; Yousef, A.; Sababheh, M. A new definition of fractional derivatuive. J. Comput. Appl. Math. 2014, 264, $65-70$.

16. Abdeljawad, T. (2015). On conformable fractional calculus. Journal of computational and Applied Mathematics, 279, 5766.

17. Zhao, D., \& Luo, M. (2017). General conformable fractional derivative and its physical interpretation. Calcolo, 54(3), 903-917.

18. Sikora, R. (2017). Fractional derivatives in electrical circuit theory-critical remarks. Archives of Electrical Engineering, 66(1), 155-163.

19. Lewandowski, M., \& Orzyłowski, M. (2017). Fractional-order models: The case study of the supercapacitor capacitance measurement. Bulletin of the Polish Academy of Sciences Technical Sciences, 65(4), 449-457.

20. Kopka, R. (2017). Estimation of supercapacitor energy storage based on fractional differential equations. Nanoscale research letters, 12(1), 636

21. Freeborn, T. J., Elwakil, A. S., \& Allagui, A. (2018, May). Supercapacitor fractional-order model discharging from polynomial time-varying currents. In 2018 IEEE International Symposium on Circuits and Systems (ISCAS) (pp. 1-5). IEEE.

22. Freeborn, T. J., Maundy, B., \& Elwakil, A. S. (2013) Measurement of supercapacitor fractional-order model parameters from voltage-excited step response. IEEE Journal on Emerging and Selected Topics in Circuits and Systems, 3(3), 367-376.

23. Tariboon, J., \& Ntouyas, S. K. (2016). Oscillation of impulsive conformable fractional differential equations. Open Mathematics, 14(1), 497-508

24. Piotrowska, E., \& Rogowski, K. (2017, October). Analysis of fractional electrical circuit using Caputo and conformable derivative definitions. In Conference on Non-integer Order Calculus and Its Applications (pp. 183-194). Springer, Cham.

25. Piotrowska, Ewa. "Analysis of fractional electrical circuit with sinusoidal input signal using Caputo and conformable derivative definitions." Poznan University of Technology Academic Journals. Electrical Engineering (2019).

26. Morales-Delgado, V. F., Gómez-Aguilar, J. F., \& TanecoHernandez, M. A. (2018). Analytical solutions of electrical circuits described by fractional conformable derivatives in Liouville-Caputo sense. AEU-International Journal of Electronics and Communications, 85, 108-117.

27. Martínez, L., Rosales, J. J., Carreño, C. A., \& Lozano, J. M. (2018). Electrical circuits described by fractional conformable derivative. International Journal of Circuit Theory and Applications, 46(5), 1091-1100.

28. Piotrowska, E. (2018, October). Analysis the conformable fractional derivative and Caputo definitions in the action of an electric circuit containing a supercapacitor. In Photonics Applications in Astronomy, Communications, Industry, and High-Energy Physics Experiments 2018 (Vol. 10808, p. 108081T). International Society for Optics and Photonics.

29. Morales-Delgado, V. F., Gómez-Aguilar, J. F., \& TanecoHernandez, M. A. (2018). Analytical solutions of electrical circuits described by fractional conformable derivatives in Liouville-Caputo sense. AEU-International Journal of Electronics and Communications, 85, 108-117.

30. Gómez-Aguilar, J. F. (2018). Fundamental solutions to electrical circuits of non-integer order via fractional derivatives with and 
Celal Bayar University Journal of Science

Volume 17, Issue 2, 2021, p 193-198

Doi: $10.18466 /$ cbayarfbe. 757813

without singular kernels. The European Physical Journal Plus, 133(5), 197.

31. https://www.wolframalpha.com/input/?i=cos\%28omega*t\%2Bphi $\% 29 * \% 28$ t $\% 5$ E $\% 28$ a- $1 \% 29 \% 29$

32. Allen R. L., and Mills D. (2004). Signal analysis: time, frequency, scale, and structure. John Wiley \& Sons. 